Gencer Sumbul, Marcela Charfuelan, Begüm Demir, Volker Markl

\title{
Bigearthnet: A Large-Scale Benchmark Archive for Remote Sensing Image Understanding
}

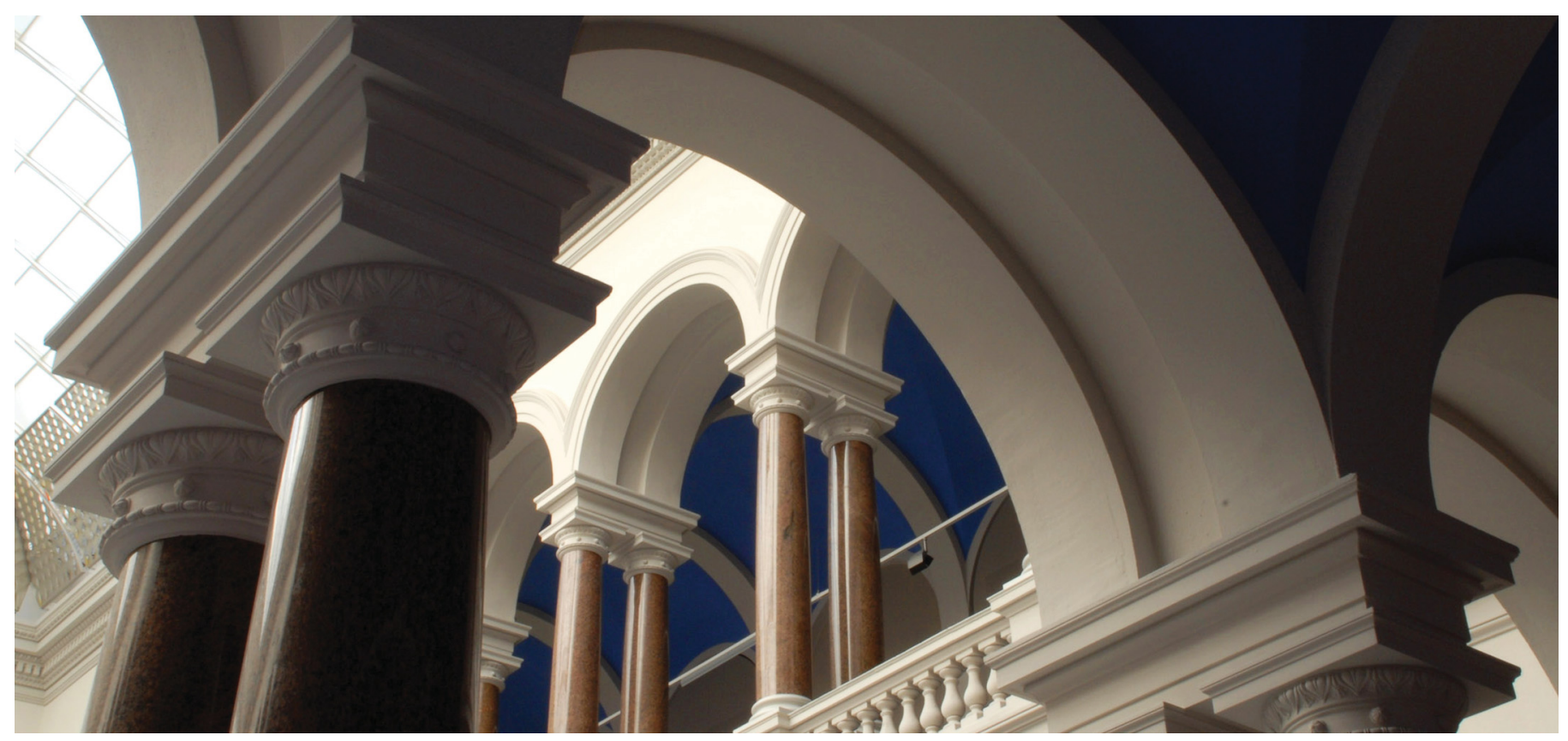

Sumbul, Gencer; Charfuelan, Marcela; Demir, Begüm; Markl, Volker (2019). Bigearthnet: A Large-Scale Benchmark Archive for Remote Sensing Image Understanding. In: IGARSS 2019 - 2019 IEEE International Geoscience and Remote Sensing Symposium. pp. 5901-5904.

https://doi.org/10.1109/IGARSS.2019.8900532 


\title{
BIGEARTHNET: A LARGE-SCALE BENCHMARK ARCHIVE FOR REMOTE SENSING IMAGE UNDERSTANDING
}

\author{
Gencer Sumbul ${ }^{1}$, Marcela Charfuelan ${ }^{2}$, Begüm Demir ${ }^{1}$, Volker Markl ${ }^{1,2}$ \\ ${ }^{1}$ Technische Universität Berlin, ${ }^{2}$ DFKI GmbH
}

\begin{abstract}
This paper presents the BigEarthNet that is a new large-scale multi-label Sentinel-2 benchmark archive. The BigEarthNet consists of 590, 326 Sentinel-2 image patches, each of which is a section of i) $120 \times 120$ pixels for $10 \mathrm{~m}$ bands; ii) $60 \times 60$ pixels for $20 \mathrm{~m}$ bands; and iii) $20 \times 20$ pixels for $60 \mathrm{~m}$ bands. Unlike most of the existing archives, each image patch is annotated by multiple land-cover classes (i.e., multi-labels) that are provided from the CORINE Land Cover database of the year 2018 (CLC 2018). The BigEarthNet is significantly larger than the existing archives in remote sensing (RS) and thus is much more convenient to be used as a training source in the context of deep learning. This paper first addresses the limitations of the existing archives and then describes the properties of the BigEarthNet. Experimental results obtained in the framework of RS image scene classification problems show that a shallow Convolutional Neural Network (CNN) architecture trained on the BigEarthNet provides much higher accuracy compared to a state-of-the-art CNN model pre-trained on the ImageNet (which is a very popular large-scale benchmark archive in computer vision). The BigEarthNet opens up promising directions to advance operational RS applications and research in massive Sentinel-2 image archives.
\end{abstract}

Index Terms - Sentinel-2 image archive, multi-label image classification, deep neural network, remote sensing

\section{INTRODUCTION}

Recent advances in deep learning have attracted great attention in remote sensing (RS) due to the high capability of deep networks (e.g., Convolutional Neural Networks (CNN), Recurrent Neural Networks (RNN), Generative Adversarial Networks (GAN)) to model the high-level semantic content of RS images. To train such networks, a very large training set is needed with a high number of annotated images in order to learn effective models with several different parameters. To the best of our knowledge, publicly available RS image archives contain only a small number of annotated images and a largescale benchmark archive does not yet exist. Thus, the lack of a large training set is an important bottleneck that prevents the use of deep learning in RS. In order to address this problem, fine-tuning deep networks pre-trained on large-scale computer vision archives (e.g., ImageNet) is considered in RS commu- nity. However, such an approach has several limitations related to the differences on the characteristics of images between computer vision and RS. Additionally, in the existing archives, RS images are annotated by single high-level category labels that are related to the most significant content of the image. However, RS images typically contain multiple classes and thus each image can be simultaneously associated with different land-cover class labels (i.e., multi-labels). To overcome these problems, we introduce the BigEarthNet that is a new large-scale Sentinel-2 archive $^{1}$ and contains 590, 326 Sentinel2 image patches. Each patch is annotated with multi-labels provided from the CORINE Land Cover database, which is updated in 2018 (CLC 2018). We propose our archive as a sufficient source for RS image analysis with deep learning. In order to test the BigEarthNet on RS image analysis problems, we focus our attention on image scene classification. To this end, we consider a shallow CNN architecture to be trained on the BigEarthNet. We compare the results obtained by this network with the Inception-v2 [1] pre-trained on the ImageNet. We believe that it will make a significant advancement in terms of developments of algorithms for the analysis of large-scale RS image archives.

\section{LIMITATIONS OF EXISTING REMOTE SENSING IMAGE ARCHIVES}

Most of the benchmark archives in RS (UC Merced Land Use Dataset [2], WHU-RS19 [3], RSSCN7 [4], SIRI-WHU [5], AID [6], NWPU-RESISC45 [7], RSI-CB [8], EuroSat [9] and PatternNet [10]) contain a small number of images annotated with single category labels. Table 1 presents the list of the existing archives. These archives become popular for the implementation, evaluation and validation of algorithms in the context of image classification, search and retrieval tasks. However, RS community encounters critical limitations, while using these archives for applying deep learning based approaches. One of the most critical limitations is that the number of annotated images included in the existing archives is very small. Thus, they are insufficient to train modern deep neural networks to reach a high generalization ability as the models may overfit dramatically when using small training sets. In details, training such networks on the existing archive images suffers from the problem of learning a large number

\footnotetext{
${ }^{1}$ The BigEarthNet is available at http://bigearth. net.
} 
Table 1: List of the existing RS archives.

\begin{tabular}{lccc}
\hline Archive Name & $\begin{array}{c}\text { Image } \\
\text { Type }\end{array}$ & $\begin{array}{c}\text { Annotation } \\
\text { Type }\end{array}$ & $\begin{array}{c}\text { Number } \\
\text { of Images }\end{array}$ \\
\hline UC Merced & Aerial RGB & $\begin{array}{c}\text { Single Label [2] } \\
\text { Multi-Label [11] }\end{array}$ & 2,100 \\
& Aerial RGB & Single Label & 1,005 \\
\hline WHU-RS19 [3] & Aerial RGB & Single Label & 2,800 \\
\hline RSSCN7 [4] & Aerial RGB & Single Label & 2,400 \\
\hline SIRI-WHU [5] & Aerial RGB & Single Label & 10,000 \\
\hline AID [6] & Aerial RGB & Single Label & 31,500 \\
\hline NWPU-RESISC45 [7] & Aerial RGB & Single Label & 36,707 \\
\hline RSI-CB [8] & Satellite Multispectral & Single Label & 27,000 \\
\hline EuroSat [9] & Aerial RGB & Single Label & 30,400 \\
\hline PatternNet [10] & & &
\end{tabular}

of parameters that prevents the accurate characterization of semantic content of RS images. To this end, fine-tuning the models pre-trained on ImageNet is used as a transfer learning approach. However, the profound differences between the image properties of computer vision and RS limit the accurate characterization of RS images when fine-tuning approach is applied. As an example, Sentinel-2 images have 13 spectral bands associated to varying and lower spatial resolutions with respect to computer vision images. There are also differences in the ways that the category labels of computer vision and RS are defined for the semantic content of an image. Thus, fine-tuning pre-trained models for RS images may not be generally applicable to reduce this semantic gap and therefore may lead to weak discrimination ability for land-cover classes. Another limitation of existing archives is that they contain images annotated by single high-level category labels, which are related to the most significant content of the image. However, RS images generally contain multiple classes so that they can be simultaneously associated to different land-cover class labels (i.e., multi-labels). Hence, a benchmark archive consisting of images annotated with multi-labels is required. Although the archive presented in [11] contains images with multi-labels, the sample size of this archive is very small to be efficiently utilized for deep learning. Another limitation of RS image archives is that since researchers generally do not have free access to satellite data together with their annotation, most of the benchmark archives contain aerial images with only RGB image bands. Unavailability of a high number of annotated satellite images prevents to employ deep learning methods in a convenient way for the complete understanding of huge amount of freely accessible satellite data (e.g., Sentinel-1, Sentinel-2). Although the benchmark archive proposed in [9] includes annotated satellite images, the number of images is still small. Aforementioned limitations of existing archives reveal the need for a large-scale RS benchmark archive to be used for training deep neural networks instead of the ImageNet.

\section{THE BIGEARTHNET ARCHIVE}

To overcome the limitations of existing archives, we introduce the BigEarthNet that is the first large-scale benchmark archive in RS. We have constructed our archive by selecting 125 Sentinel-2 tiles acquired between June 2017 and May
Table 2: The considered Level-3 CLC classes and the number of images associated with each land-cover class in the BigEarthNet.

\begin{tabular}{lc}
\hline Land-Cover Classes & $\begin{array}{c}\text { Number of } \\
\text { Images }\end{array}$ \\
\hline Mixed forest & 217,119 \\
\hline Coniferous forest & 211,703 \\
\hline Non-irrigated arable land & 196,695 \\
\hline Transitional woodland/shrub & 173,506 \\
\hline Broad-leaved forest & 150,944 \\
\hline Land principally occupied by agriculture, & 147,095 \\
\hline with significant areas of natural vegetation & 107,786 \\
\hline Complex cultivation patterns & 103,554 \\
\hline Pastures & 83,811 \\
\hline Water bodies & 81,612 \\
\hline Sea and ocean & 69,872 \\
\hline Discontinuous urban fabric & 30,674 \\
\hline Agro-forestry areas & 23,207 \\
\hline Peatbogs & 13589 \\
\hline Permanently irrigated land & 12895 \\
\hline Industrial or commercial units & 12,835 \\
\hline Natural grassland & 12,538 \\
\hline Olive groves & 11,241 \\
\hline Sclerophyllous vegetation & 10,784 \\
\hline Continuous urban fabric & 10,572 \\
\hline Water courses & 9,567 \\
\hline Vineyards & 7,022 \\
\hline Annual crops associated with permanent crops & 6,236 \\
\hline Inland marshes & 5,890 \\
\hline Moors and heathland & 5,353 \\
\hline Sport and leisure facilities & 4,754 \\
\hline Fruit trees and berry plantations & 4,618 \\
\hline Mineral extraction sites & 3,793 \\
\hline Rice fields & 3,384 \\
\hline Road and rail networks and associated land & 3,277 \\
\hline Bare rock & 1,786 \\
\hline Green urban areas & 1,578 \\
\hline Beaches, dunes, sands & 1,563 \\
\hline Sparsely vegetated areas & 1,562 \\
\hline Salt marshes & 1,498 \\
\hline Coastal lagoons & 1,174 \\
\hline Construction sites & 1,086 \\
\hline Estuaries & 1,003 \\
\hline Intertidal flats & 979 \\
\hline Airports & 959 \\
\hline Dump sites & 509 \\
\hline Port areas & 424 \\
\hline Salines & 328 \\
\hline Burnt areas & \\
\hline \hline & \\
\hline &
\end{tabular}

2018. Considered tiles are distributed over the 10 countries (Austria, Belgium, Finland, Ireland, Kosovo, Lithuania, Luxembourg, Portugal, Serbia, Switzerland) of Europe. It is worth noting that considered tiles are associated to cloud cover percentage less than $1 \%$. All tiles were atmospherically corrected by using Sentinel-2 Level 2A product generation and formatting tool (sen2cor) of ESA. Among 13 Sentinel-2 spectral bands, $10^{\text {th }}$ band, for which surface information is not embodied, was excluded. After the tile selection and preliminary processing steps were carried out, selected tiles were divided into 590, 326 non-overlapping image patches. Each patch (denoted as image hereafter) is a section of i) $120 \times 120$ pixels for $10 \mathrm{~m}$ bands; ii) $60 \times 60$ pixels for $20 \mathrm{~m}$ bands; and iii) $20 \times 20$ pixels for $60 \mathrm{~m}$ bands. We have associated each image with one or more land-cover class labels (i.e., multi-labels) provided from the CORINE Land Cover (CLC) database of the year 2018 (CLC 2018). The CLC inventory was produced by the Eionet National Reference Centres on Land Cover with the 

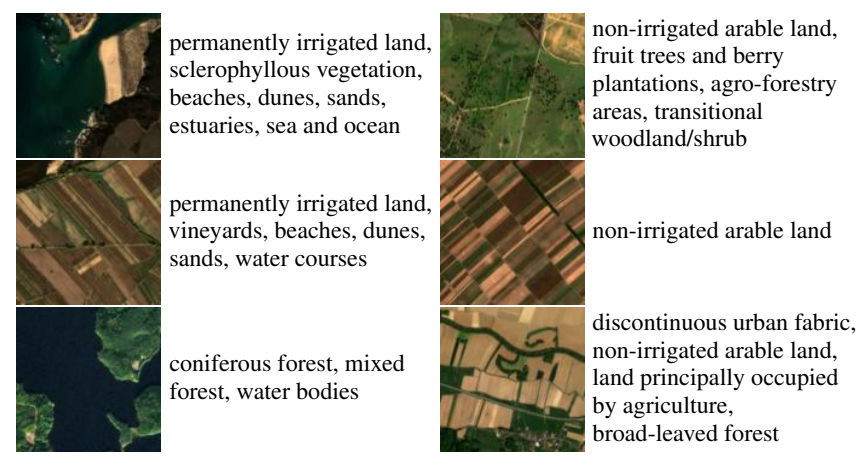

Fig. 1: Example of Sentinel-2 images and their multi-labels in our BigEarthNet archive.

coordination of the European Environment Agency (EEA) for the recognition, identification and assessment of land cover classes by leveraging the texture, pattern and density information of the objects presented in RS images. This inventory is very recently updated as CLC 2018, for which the annotation process has been carried out for the period of 2017-2018. We selected tiles within the considered time interval to be appropriate for the annotation period of CLC 2018. CLC nomenclature includes land cover classes grouped in a three-level hierarchy ${ }^{2}$. The considered Level-3 CLC class labels and the number of images associated with each label are shown in Table 2. We would like to note that the number of images for each land cover class varies significantly in the archive. The number of labels associated with each image varies between 1 and 12, whereas $95 \%$ of images have at most 5 multi-labels. Only 15 images contain more than 9 labels in the BigEarthNet. Fig. 1 shows an example of images and their multi-labels, while Fig. 2 shows the number of Sentinel-2 images with respect to the acquisition date. It is worth noting that we aimed to represent each considered geographic location with images acquired in all different seasons. However, due to the difficulties of collecting Sentinel-2 images with lower cloud cover percentage within a narrow time interval, it was not possible for some areas. The number of images acquired in autumn, winter, spring and summer seasons are 154943, 117156, 189276 and 128951 respectively. Since cloud cover percentage of Sentinel-2 tiles acquired in winter is generally higher than the other seasons, our archive contains the lowest number of images from winter season.

We also employed the visual inspection for the quality check of image multi-labels. By visual inspection, we have identified that 70,987 images are fully covered by seasonal snow, cloud and cloud shadow ${ }^{3}$. We suggest not to include these images for training and test stages of the machine/deep learning algorithms, while working on scene classification, content-based image retrieval and search if only BigEarthNet Sentinel-2 images are used.

\footnotetext{
${ }^{2}$ https: //land.copernicus.eu/user-corner/ technical-library/corine-land-cover-nomenclatureguidelines

${ }^{3}$ The lists of images fully covered by seasonal snow, cloud and cloud shadow are available at http://bigearth. net/\#downloads.
}

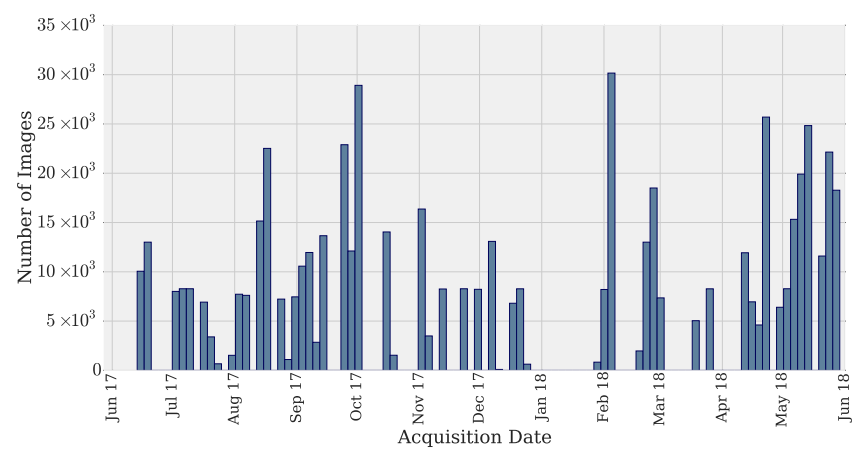

Fig. 2: The number of Sentinel-2 images with respect to acquisition date.

\section{EXPERIMENTAL RESULTS}

In the experiments, we have used the BigEarthNet archive in the framework of RS image scene classification problems. To this end, we selected a shallow CNN architecture, which consists of three convolutional layers with 32,32 and 64 filters having $5 \times 5,5 \times 5$ and $3 \times 3$ filter sizes, respectively. We added one fully connected (FC) layer and one classification layer to the output of last convolutional layer. In all convolution operations, zero padding was used. We also applied max-pooling between layers. We considered to utilize: i) only RGB channels (denoted as S-CNN-RGB); and ii) all spectral channels (denoted as S-CNN-All). For the S-CNN-All, cubic interpolation was applied to 20 and 60 meter bands of each image to have the same pixel sizes associated with each band. Weights of the S-CNN-RGB and the S-CNN-All were randomly initialized and we trained both networks from scratch on the BigEarthNet images. In order to show the effectiveness of the BigEarthNet to be used in training, we compared the results with fine-tuning one of the recent pre-trained deep learning architectures. We considered the Inception-v2 network [1] pre-trained on ImageNet as a state-of-the-art architecture. We used the feature vector extracted from the layer just before the softmax layer of the Inception-v2. To employ finetuning, we fixed the model weights of the Inception network. We added one FC and one classification layer to the network and just fine-tuned these layers by using the RGB channels of the BigEarthNet images. In the experiments, 70, $987 \mathrm{im}$ ages that are fully covered by seasonal snow, cloud and cloud shadow were eliminated. Then, among the remaining images, we randomly selected: i) $60 \%$ of images to derive a training set; ii) $20 \%$ of images to derive a validation set; and iii) $20 \%$ of images to derive a test set. Both for fine-tuning and training from scratch, we selected the number of epochs as 100 and Stochastic Gradient Descent algorithm is employed in order to decrease the sigmoid cross entropy loss (which aims at maximizing the log-likelihood of each land-cover class throughout all training images). For the performance metrics of experiments, we employed precision $(P)$, recall $(R), F 1$ and $F 2$ scores, which are widely used metrics for multi-label image classification. As it can be seen from Table 4, the S-CNN-RGB provides better performance than the Inception-v2 in all met- 
Table 3: Example of Sentinel-2 images with the true multi-labels and the multi-labels assigned by the Inception-v2, the S-CNN-RGB and the S-CNN-All.

\begin{tabular}{|c|c|c|c|c|}
\hline Test Images & True Multi-Label & Inception-v2 & S-CNN-RGB & S-CNN-All \\
\hline & pastures, peatbogs & $\begin{array}{l}\text { non-irrigated arable land, } \\
\text { coniferous forest, mixed forest, } \\
\text { transitional woodland/shrub }\end{array}$ & $\begin{array}{l}\text { non-irrigated arable land, land } \\
\text { occupied by agriculture, mixed } \\
\text { forest }\end{array}$ & pastures, peatbogs \\
\hline & $\begin{array}{l}\text { pastures, land occupied } \\
\text { by agriculture, water } \\
\text { bodies }\end{array}$ & $\begin{array}{l}\text { coniferous forest, mixed } \\
\text { forest, transitional } \\
\text { woodland/shrub }\end{array}$ & $\begin{array}{l}\text { non-irrigated arable land, } \\
\text { land occupied by } \\
\text { agriculture }\end{array}$ & $\begin{array}{l}\text { pastures, land occupied } \\
\text { by agriculture, water } \\
\text { bodies }\end{array}$ \\
\hline & $\begin{array}{l}\text { discontinuous urban } \\
\text { fabric, industrial or } \\
\text { commercial units }\end{array}$ & $\begin{array}{l}\text { coniferous forest, mixed } \\
\text { forest, transitional } \\
\text { woodland/shrub }\end{array}$ & $\begin{array}{c}\text { discontinuous urban fabric, land occupied } \\
\text { by agriculture, broad-leaved forest, } \\
\text { coniferous forest, mixed forest }\end{array}$ & $\begin{array}{l}\text { discontinuous urban } \\
\text { fabric, industrial or } \\
\text { commercial units }\end{array}$ \\
\hline
\end{tabular}

Table 4: Experimental results obtained by the Inception-v2, the SCNN-RGB and the S-CNN-All.

\begin{tabular}{lcccc}
\hline Method & $P(\%)$ & $R(\%)$ & $F_{1}$ & $F_{2}$ \\
\hline Inception-v2 [1] & 48.23 & 56.79 & 0.4988 & 0.5301 \\
S-CNN-RGB & 65.06 & 75.57 & 0.6759 & 0.7139 \\
S-CNN-All & $\mathbf{6 9 . 9 3}$ & $\mathbf{7 7 . 1 0}$ & $\mathbf{0 . 7 0 9 8}$ & $\mathbf{0 . 7 3 8 4}$ \\
\hline
\end{tabular}

rics, while both networks consider only RGB image channels. When the S-CNN-All architecture is trained on the BigEarthNet images containing all spectral bands, the results become much more promising with respect to using only RGB bands. Table 3 shows the example of Sentinel-2 images with the true multi-labels and the multi-labels assigned by the Inceptionv2, the S-CNN-RGB and the S-CNN-All. The performance improvements on all metrics are statistically significant under a value of $p \ll 0.0001$. The same behavior is also observed when the BigEarthnet images are associated to Level-1 and Level-2 CLC class labels. We would like to also note that the S-CNN-RGB and the S-CNN-All are very simple CNN architectures that consist of only 3 convolutional layers and max-pooling. Training deeper models (which include recent deep learning techniques such as residual connections, wider layers with varying filter sizes etc.) from scratch can lead to more promising results. On the basis of all obtained results, we can state that RS community can benefit from these pretrained models on the BigEarthNet instead of the computer vision archives.

\section{CONCLUSION}

This paper presents a large-scale benchmark archive that consists of 590,326 Sentinel-2 image patches annotated by multi-labels for RS image understanding. We believe that the BigEarthNet will make a significant advancement for the use of deep learning in RS by overcoming the current limitations of the existing archives. Experimental results show the effectiveness of training even a simple neural network on the BigEarthNet from scratch compared to fine-tuning a stateof-the-art deep learning model pre-trained on the ImageNet. We would like to note that we plan to regularly enrich the BigEarthNet by increasing the number of annotated Sentinel-2 images.

\section{ACKNOWLEDGEMENTS}

This work was supported by the European Research Council under the ERC Starting Grant BigEarth (759764) and the German Ministry for Education and Research as BBDC (01IS14013A).

\section{REFERENCES}

[1] C. Szegedy, V. Vanhoucke, S. Ioffe, J. Shlens, and Z. Wojna, "Rethinking the inception architecture for computer vision," in IEEE Conf. Comput. Vis. Pattern Recog., 2016.

[2] Y. Yang and S. Newsam, "Bag-of-visual-words and spatial extensions for land-use classification," in Intl. Conf. Adv. Geogr. Inf. Syst., 2010.

[3] W. Shao, W. Yang, and G. S. Xia, "Extreme value theory-based calibration for the fusion of multiple features in high-resolution satellite scene classification," Int. J. Remote Sens., vol. 34, no. 23, pp. 8588-8602, 2013.

[4] Q. Zou, L. Ni, T. Zhang, and Q. Wang, "Deep learning based feature selection for remote sensing scene classification," IEEE Geosci. Remote Sens. Lett., vol. 12, no. 11, pp. 2321-2325, November 2015.

[5] B. Zhao, Y. Zhong, G. Xia, and L. Zhang, "Dirichlet-derived multiple topic scene classification model for high spatial resolution remote sensing imagery," IEEE Trans. Geosci. Remote Sens., vol. 54, no. 4, pp. 2108-2123, April 2016.

[6] G. Xia, J. Hu, F. Hu, B. Shi, X. Bai, Y. Zhong, L. Zhang, and X. Lu, "Aid: A benchmark data set for performance evaluation of aerial scene classification," IEEE Trans. Geosci. Remote Sens., vol. 55, no. 7, pp. 3965-3981, July 2017.

[7] G. Cheng, J. Han, and X. Lu, "Remote sensing image scene classification: Benchmark and state of the art," Proc. IEEE, vol. 105, no. 10, pp. 1865-1883, October 2017.

[8] H. Li, C. Tao, Z. Wu, J. Chen, J. Gong, and M. Deng, "Rsi-cb: A large scale remote sensing image classification benchmark via crowdsource data," arXiv preprint arXiv:1705.10450, 2017.

[9] P. Helber, B. Bischke, A. Dengel, and D. Borth, "Eurosat: A novel dataset and deep learning benchmark for land use and land cover classification," arXiv preprint arXiv:1709.00029, 2017.

[10] W. Zhou, S. Newsam, C. Li, and Z. Shao, "Patternnet: A benchmark dataset for performance evaluation of remote sensing image retrieval," ISPRS J. Photogram. Remote Sens., vol. 145, pp. 197-209, 2018.

[11] B. Chaudhuri, B. Demir, S. Chaudhuri, and L. Bruzzone, "Multilabel remote sensing image retrieval using a semisupervised graph-theoretic method," IEEE Trans. Geosci. Remote Sens., vol. 56, no. 2, pp. 1144-1158, February 2018. 